\title{
GOP contribution to independent monitoring of Galileo OS navigation performance
}

Douša Jan ${ }^{1}$, Václavovic Pavel ${ }^{1}$, Michael Kala ${ }^{1}$, Petr Bezděka ${ }^{1}$, Lewen Zhao ${ }^{1,2}$

${ }^{1}$ Research Institute of Geodesy, Topography and Cartography, Zdiby, 25066, Czech Republic

${ }^{2}$ School of Remote Sensing and Geomatics Engineering, Nanjing University of Information Science and Technology, Nanjing, 210044, China

Correspondence to: Jan Douša1 (jan.dousa@pecny.cz)

https://doi.org/10.31490/9788024845050-6

Abstract

Geodetic Observatory Pecný (GOP) of the Research Institute of Geodesy, Topography and Cartography contributes to the monitoring navigation performance of the Galileo Open Service Signal in Space within the Member States support of the Galileo Reference Centre. Our contribution consists of the three main tasks: 1) monitoring the quality of multi-GNSS data stemming from 65 global stations, 2) generating consolidated navigation files and reference GPS and Galileo satellites orbit and clock products with a latency of 6 and 42 hours, and 3) estimating key-parameter indicators for the Galileo OS navigation performance monitoring. The GOP chain of monitoring processes and all mandatory inputs is independent from other contributions in terms of the tools (G-Nut software), reference products (GOP rapid precise satellite orbits and clocks), and consolidated navigation data (GOP BRDC files).

Keywords: GNSS, precise orbits and clocks, PPP, navigation data and performance

\section{INTRODUCTION}

Since November 2018, the Geodetic Observatory Pecný (GOP) of the Research Institute of Geodesy, Topography and Cartography has contributed to the monitoring navigation performance of the Galileo Open Service Signal in Space (OS SIS). The activity is performed within the Member States support (GRC-MS project) to the Galileo Reference Centre (GRC), in 2018 opened by the European GNSS Agency (GSA) in Noordwijk, the Netherlands.

The GOP contributes to the GRC-MS with the following tasks:

- Monitoring the quality of multi-GNSS data from about 65 global GRC-MS stations.

- Providing early-rapid and rapid reference products:

- Consolidated navigation data (BRDC files)

- Precise GPS and Galileo orbits and clock corrections for active satellites

- Monitoring the performance of the Galileo OS navigation:

- Service volume availability of at least one dual-frequency Galileo OS SIS

- Service volume availability of PDOP $<6$

- Horizontal and vertical positioning service accuracy 
The paper describes individual activities and tasks, see Figure 1 which shows all the contributions in a single chart for visualizing a complete chain of all performance monitoring processes. The most important is that these are independent in terms of the software (the G-Nut software, the Bernese software is an exception for the orbit determination) as well as mandatory inputs, in our case the GOP reference products provided in a rapid mode with a maximum delay of 48 hours.

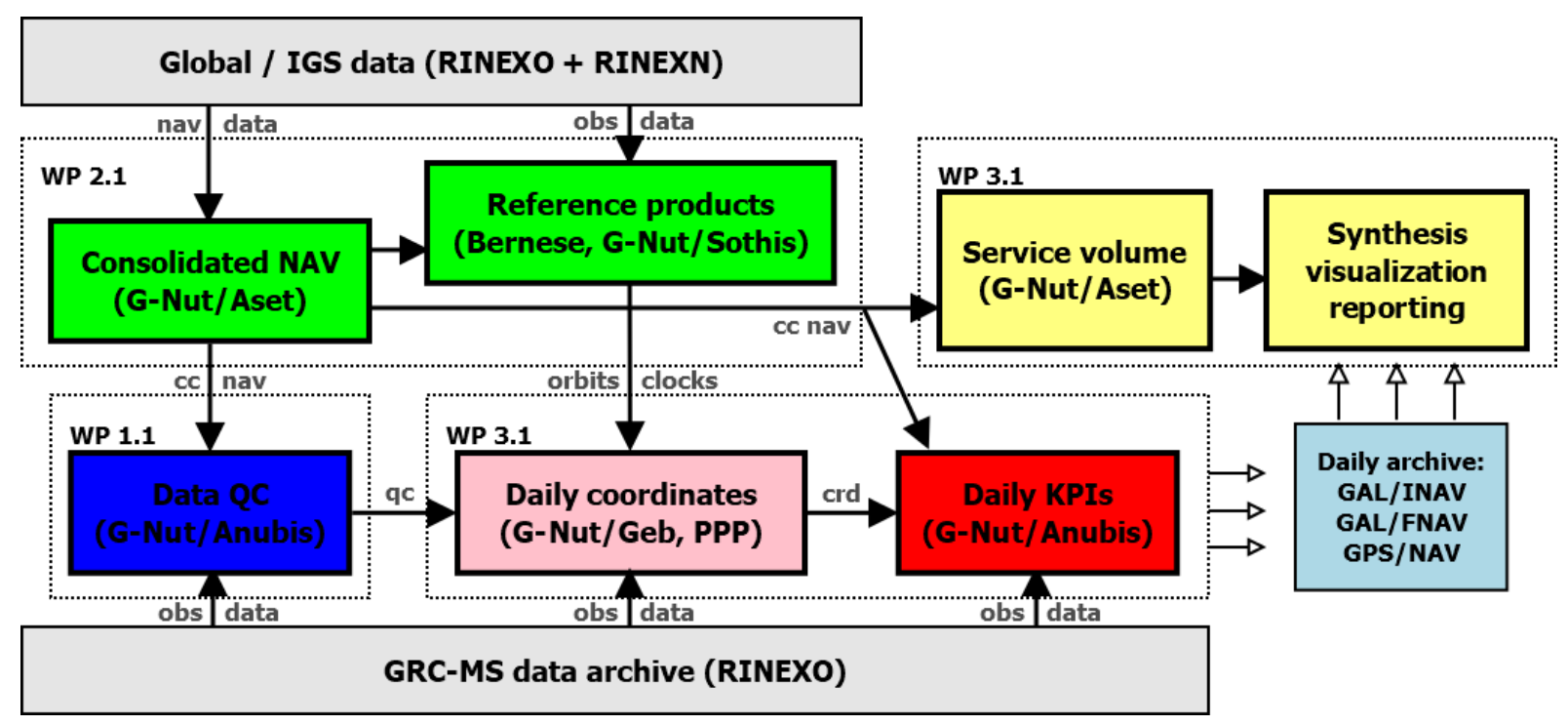

Fig. 1. Chart of independent chain of the monitoring procedures, inputs and software

\section{CONSOLIDATED NAVIGATION DATA (BRDC FILES)}

The GOP consolidated navigation BRDC files (Douša 2019) are created using the GNut/Aset software from real-time streams and hourly/daily RINEX files of 300+ global multiGNSS stations of the IGS (Dow et al. 2009), MGEX (Montenbruck et al. 2017) and GRCMS networks. The product contains navigation data for all GNSS constellations and regional augmentations. The RINEX 2 and 3 files are provided repetitively over past three days with a delay of 2-72 hours.

The GOP consolidation procedure consists of merging, filtering, and data quality control of navigation records collected from a large number of global multi-GNSS stations. It requires neither a priori information nor external reference products for the ultra-fast delivery besides relaying on several autonomous methods to guarantee a high reliability: a) time-series analysis for selected parameters (values, differences), b) range check (record pre-filtering), c) statistical analysis, d) majority vote, e) penalty system, f) identification and correction of issues from specific receivers.

The parameter range check is used for a simple filtering of (obviously) incorrect navigation records. A time-series analysis checks absolute values (and their changes in time) for selected navigation parameters which is completed with a penalty system using adaptable thresholds for identifying and rejecting outliers. Signal group delays and satellite health status are evaluated statistically along with autonomously detecting (and eliminating) problematic receiver types. Header data are selected according to a majority vote. All the methods applied within the concatenation and the quality-control processes are performed 
at several levels when selecting group of navigation files from global stations.

Figure 2 summarizes a total number of satellites per individual GNSS and mean number of navigation messages per satellite for each GNSS as available from the GOP BRDC during the $4^{\text {th }}$ quarter of 2020 . Figure 3 then displays a daily percentage of healthy FNAV (Galileo) and NAV (GPS) navigation records per day for individual satellites (indicated on the vertical axis). The value $100 \%$ and $0 \%$ represents active and unhealthy satellites, respectively. Some days/satellites show records mixing healthy and unhealthy signal status. Note that Galileo health status is combined from the SHS, DVS and SISA indicators as defined in the Galileo Initial Services - Open Service SDD (GSA 2019). The plots show two Galileo eccentric satellites (E14 and E18) becoming active on November 30, 2020 (NAGU 202001920). Since that time, the Galileo space segment consists of 24 active satellites and 2 (permanently) inactive satellites. An event occurred on December 14, 2020, when Galileo FNAV and INAV OS SIS was not available during 4 and 6 hours (NAGU 2020021), respectively, due to the SISA=NAPA as clearly visible in Fig. 4.
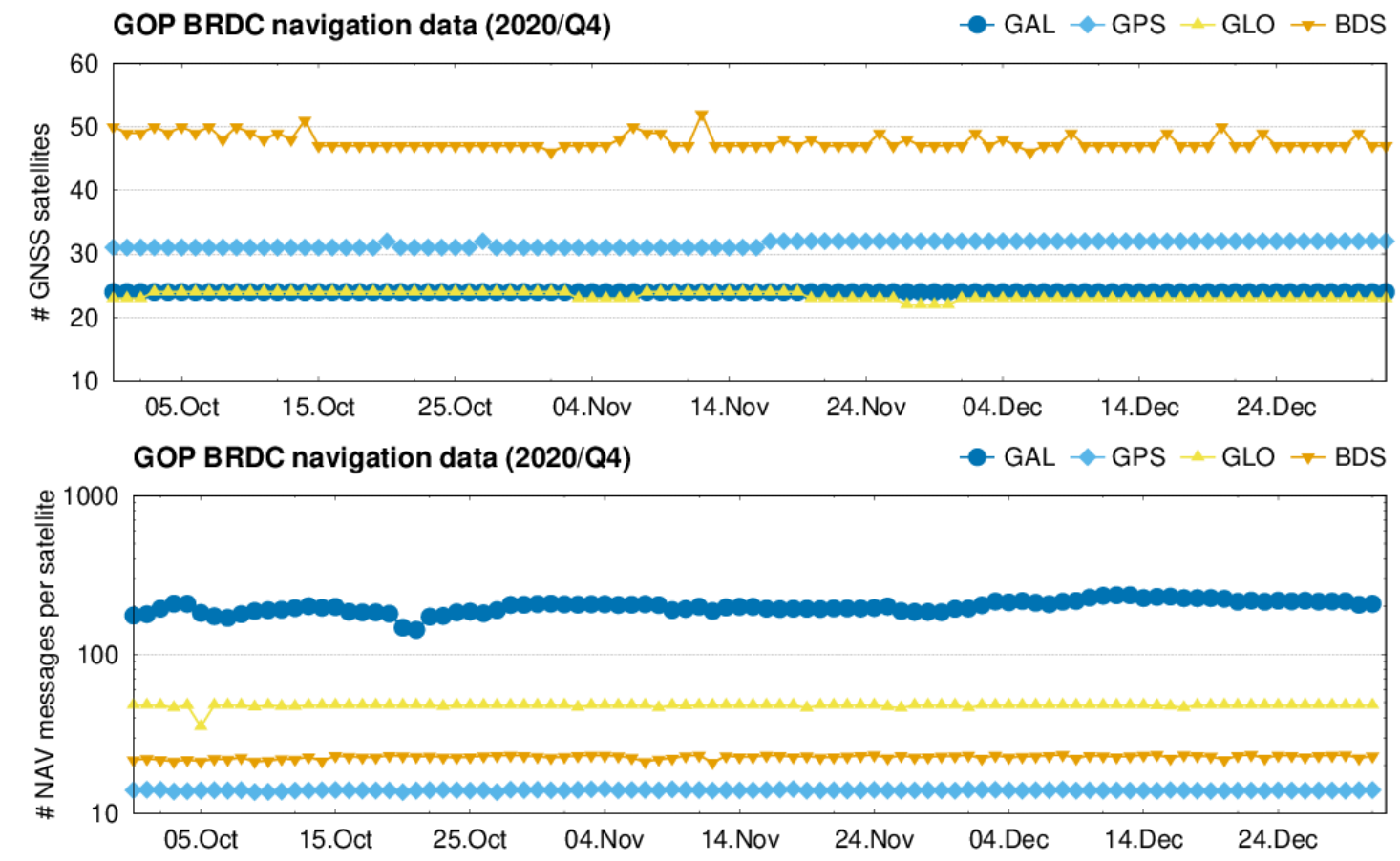

Fig. 2. Number of satellites per system (top), mean number of navigation records per satellite (bottom) 
GAL - satellite health status [FNAV]

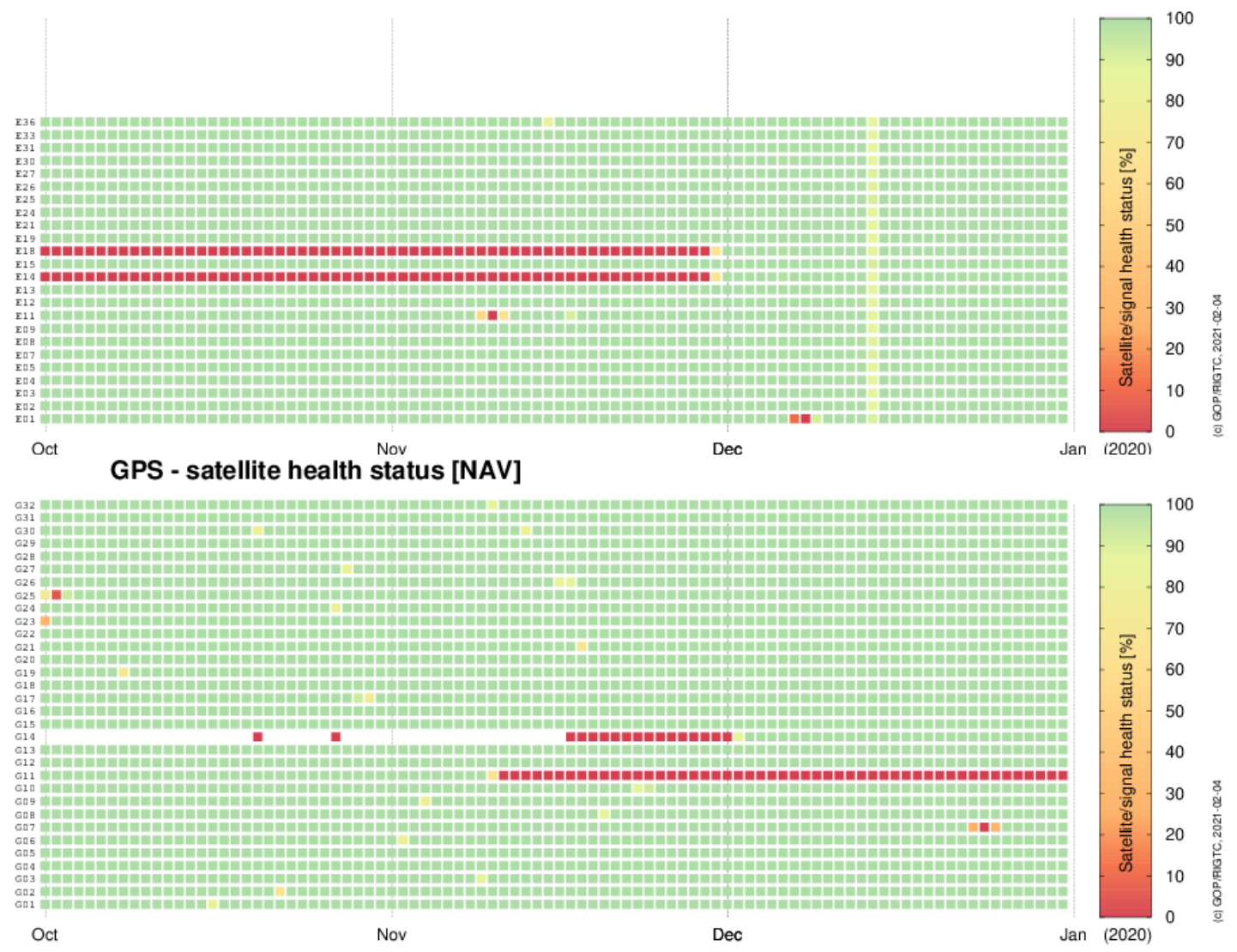

Fig. 3. Daily percentages of FNAV and NAV navigation records of Galileo (top) and GPS (bottom), respectively; $Y$-axis represents to individual satellites

GAL satellite - Satellite SISA [GOP BRDC, FNAV type]

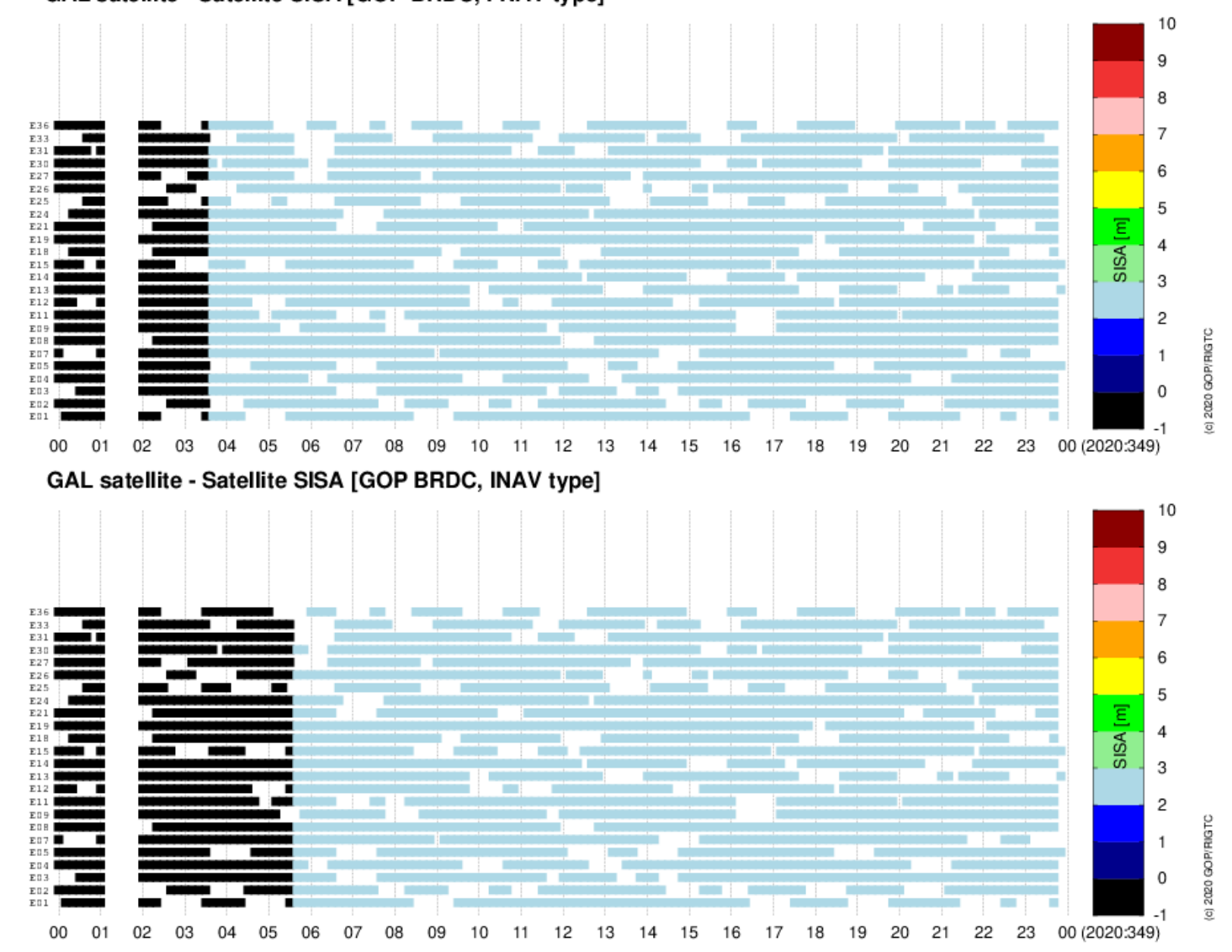

Fig. 4. Galileo SISA values on Dec 14, 2020 for FNAV (top) and INAV (bottom) records, $x$-axis: ToC 


\section{REFERENCE SATELLITE ORBIT AND CLOCK PRODUCTS}

The GOP uses the Bernese GNSS Software V5.2 software (Dach et al. 2020) for generating precise orbits exploiting double-difference observations from a network of $120+$ global stations. The processing strategy was derived from the GOP ultra-rapid orbits procedure contributing to IGS (Douša 2004, 2012), however, now including the Galileo besides GPS system. Two solutions are generated: 1) rapid with a latency of 2 days, and 2) early-rapid with a latency of 6 hours. The latter is a trade-off between IGS standard ultra-rapid and rapid production, generated once a day, but including 1-day orbit prediction too.

The orbit product results from combining two solutions from consecutive days, i.e. exploiting a two-day orbit arc solution. The solution includes estimating 6 Kepler orbital parameters, 9 solar radiation pressure parameters of the extended CODE model V2 (ECOM2, Arnold et al. 2015), and completed with 3 stochastic pulses for every 12 hours. The orbit determination is initialized exploiting GOP BRDC files. The procedure then consists of two main iterations while others may be triggered anytime when handling specific problems due to individual satellites and stations. Satellite-specific accuracy codes in the SP3 header are estimated by exploiting variety of information, e.g. parameter formal errors, day-to-day orbit comparisons, short-/long-arc combinations, as well as other internal control procedures.

Station coordinates and Earth rotation parameters (X-/Y-Pole, X-/Y-Pole rates, LOD) are estimated along with the satellite orbits on a daily basis too. Other parameters, such as tropospheric delays, ionospheric corrections, initial phase ambiguities, are handled within individual processing steps with a higher temporal resolution. The models used in the processing are compliant with the IERS2010 conventions. The absolute antenna type calibrations for phase centre offsets and variations are regularly updated from the IGS14 model. The DLR MGEX quarterly biases (Montenbruck et al. 2014) are used for correcting satellite differential code biases within the processing.

The GOP precise satellite clock corrections are estimated on a daily basis using the GNut/Sothis software while introducing GOP early-rapid (or rapid) satellite orbits and station coordinates. The processing strategy also exploits the ionosphere-free linear combination, however, mixing zero/epoch-difference observations. Epoch clock variations are estimated by using epoch-differenced carrier-phase observations and initial clock biases (ICBs) for each individual satellite are estimated by using zero-differenced code observations (Ge et al. 2012). The method is both efficient and robust thanks to the eliminating initial phase ambiguities and reducing any possible negative impact of cycle slips. Satellite and receiver clock corrections are estimated epoch-by-epoch in a stochastic process (the Kalman filter) as a sum of epoch-differenced clock corrections and the corresponding initial clock bias. For accurate ICB estimates, the procedure is supported with differential code biases from DLR. The clock datum is defined using the common clock satellite datum with the strategy developed at GOP particularly for real-time clock estimates (Zhao et al. 2020). The clock corrections are estimated at a 5-min sampling rate for all healthy GPS and Galileo satellites, and these are provided consistently in both SP3 and clock RINEX files.

We compare regularly the GOP orbits and clocks with respect to the IGS MGEX products, in particular CODE (Prange et al. 2015, 2017) and CNES solutions (Loyer et al. 2018). The 
comparison is performed on a daily basis using the G-Nut/Aset software and a 15-min time resolution. The orbit comparison includes the calculation of 7 Helmert parameters between both solutions in a global scale and daily basis when removing problematic satellites (if any). The satellite clock comparisons is performed on a double-difference basis, first, for eliminating the clock datum definition within individual products (accessible as a common clock error in a single epoch) and, second, for eliminating initial satellite clock biases which correspond to satellite-specific code biases at the initial epoch. The clock differences are also reduced by the radial position error (for this case transformed into time domain by applying the speed of light) for the corresponding satellite, because the actual clocks also include the compensation of errors in the radial component.

Figure 5 and Figure 6 show results of comparisons of Galileo and GPS satellite orbits and clock corrections, respectively, between GOP Rapid and CODE Final products. In long-term, the GOP rapid orbits agree to CODE and CNES final products with a 3D RMS of $3 \mathrm{~cm}$ and $6 \mathrm{~cm}$ for GPS and Galileo, respectively. The two Galileo elliptic satellites were included in GOP solution immediately when became active on November 30, 2020.

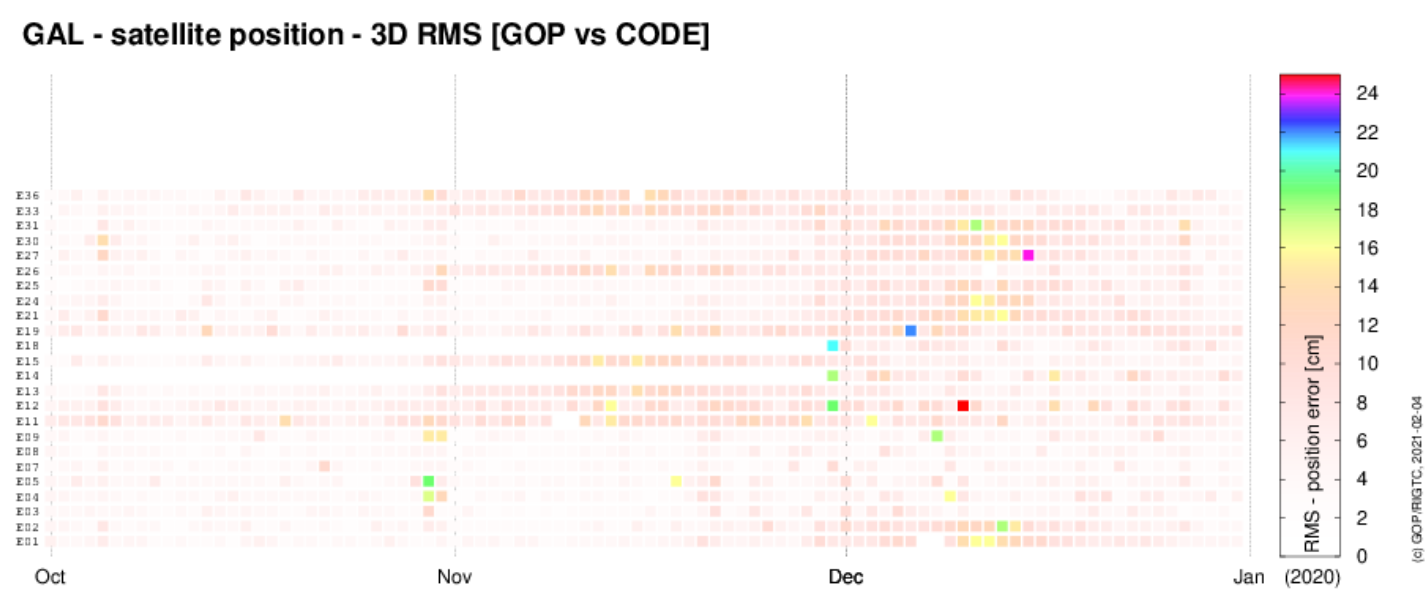

GAL - satellite clocks [GOP vs CODE]

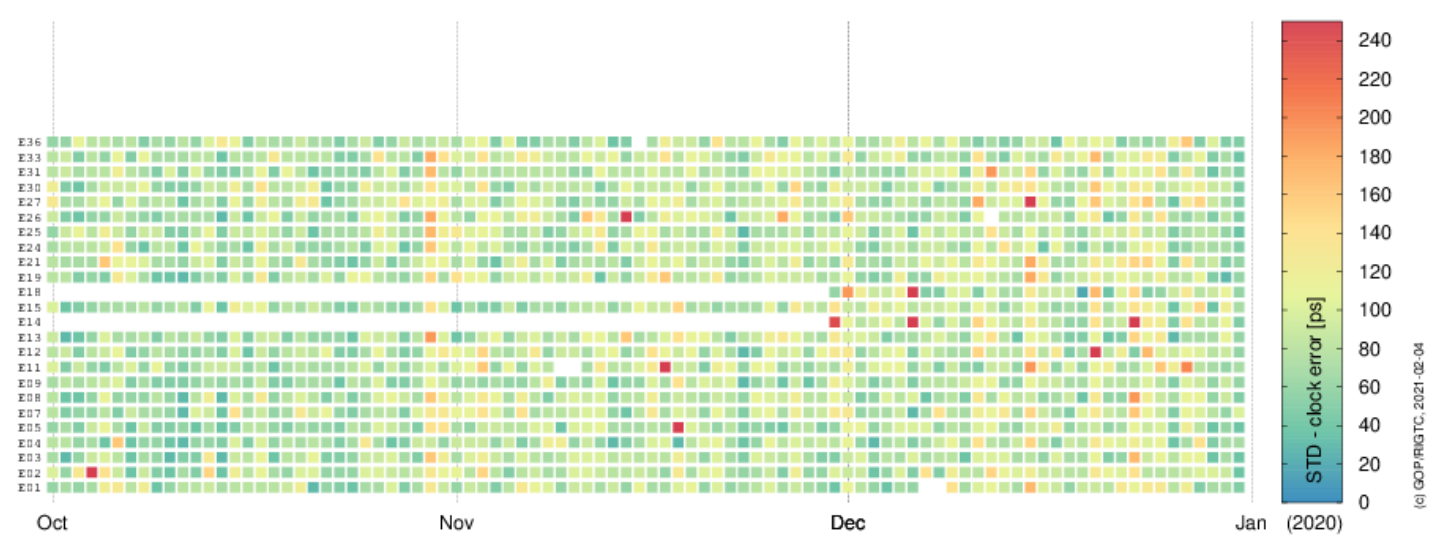

Fig. 5. Comparison of GOP rapid vs. CODE final orbit (top) and clock (bottom) products: Galileo system 


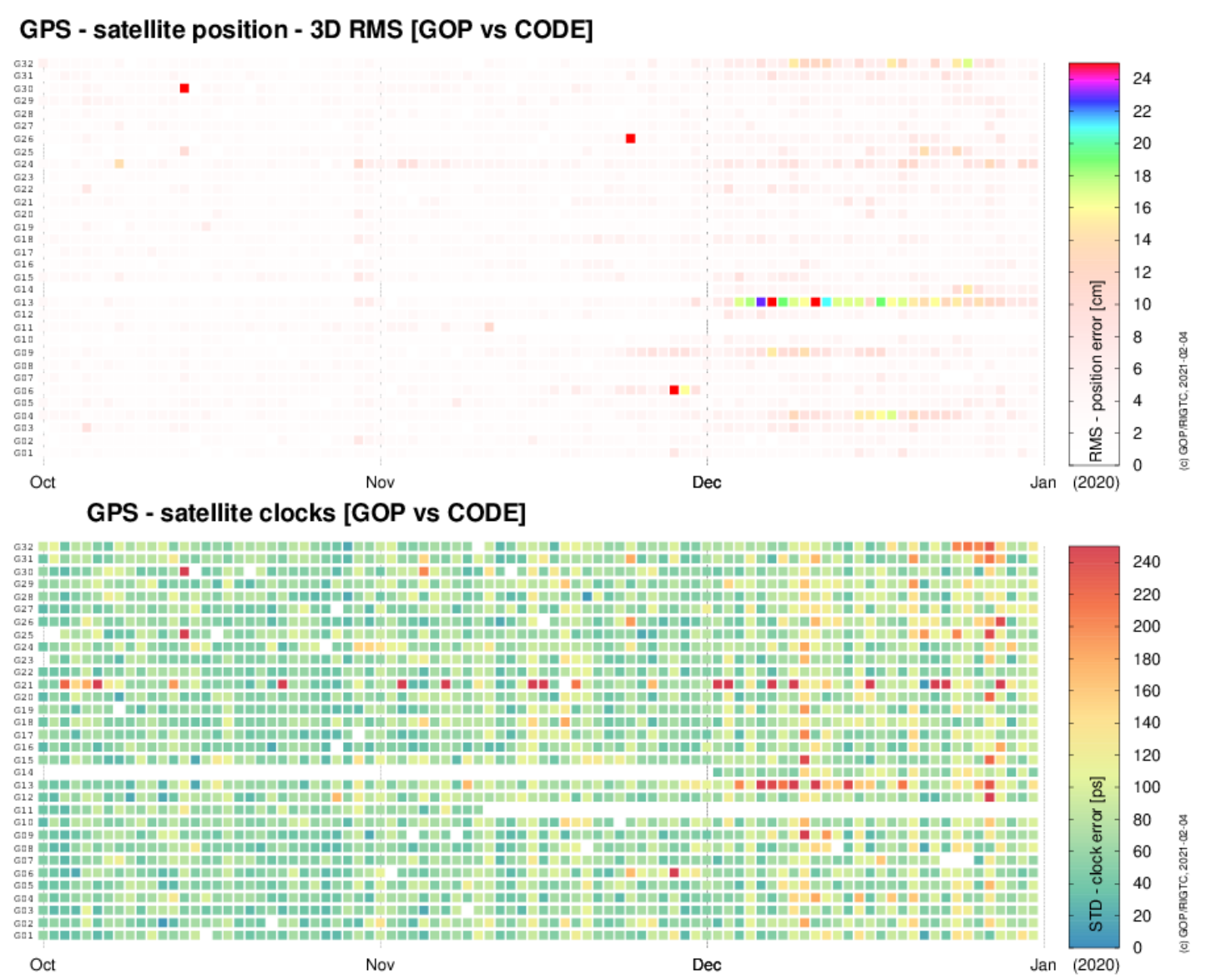

Fig. 6. Comparison of GOP rapid vs. CODE final orbit (left) and clock (right) products: GPS system

\section{AVAILABILITY OF GLOBAL 3D DILUTION OF PRECISION (PDOP < 6 )}

The availability of the Galileo global 3D Dilution of Precision is monitored using a monthly set of GOP BRDC files. A service volume monitoring is addressed using a regular grid of 'user' points with a horizontal resolution of $10 \times 10$ degrees, and the 900 seconds sampling interval. For each grid point and time epoch, the Position DOP (PDOP) is calculated from all healthy satellites (considering SHS, DVS, SISA and the ephemeris validity period) above the elevation angle cut-off 5 degrees. The availability of PDOP $<6$ is then estimated using the G-Nut/Aset software on a monthly basis as a weighted (over latitudes) mean values.

In February 11, 2019, when in total 22 Galileo satellites became active, the Initial Service achieved globally stable and low PDOP values which enabled a continuous positioning at any place on the Earth over all days. The monthly mean availability of PDOP $<6$ became better than $99 \%$, with an exception of $87 \%$ occurring in July 2019 . The Galileo OS-SIS SDD requirement defines $78 \%$ which was satisfied all the time during 2019 and 2020 years).

Figure 7 displays global maps of the mean availability of the Galileo 3D dilution of precision (PDOP<6) for November and December 2020 for FNAV (left) and INAV (right) data types. The latter includes the abovementioned event on December 14, 2020. 

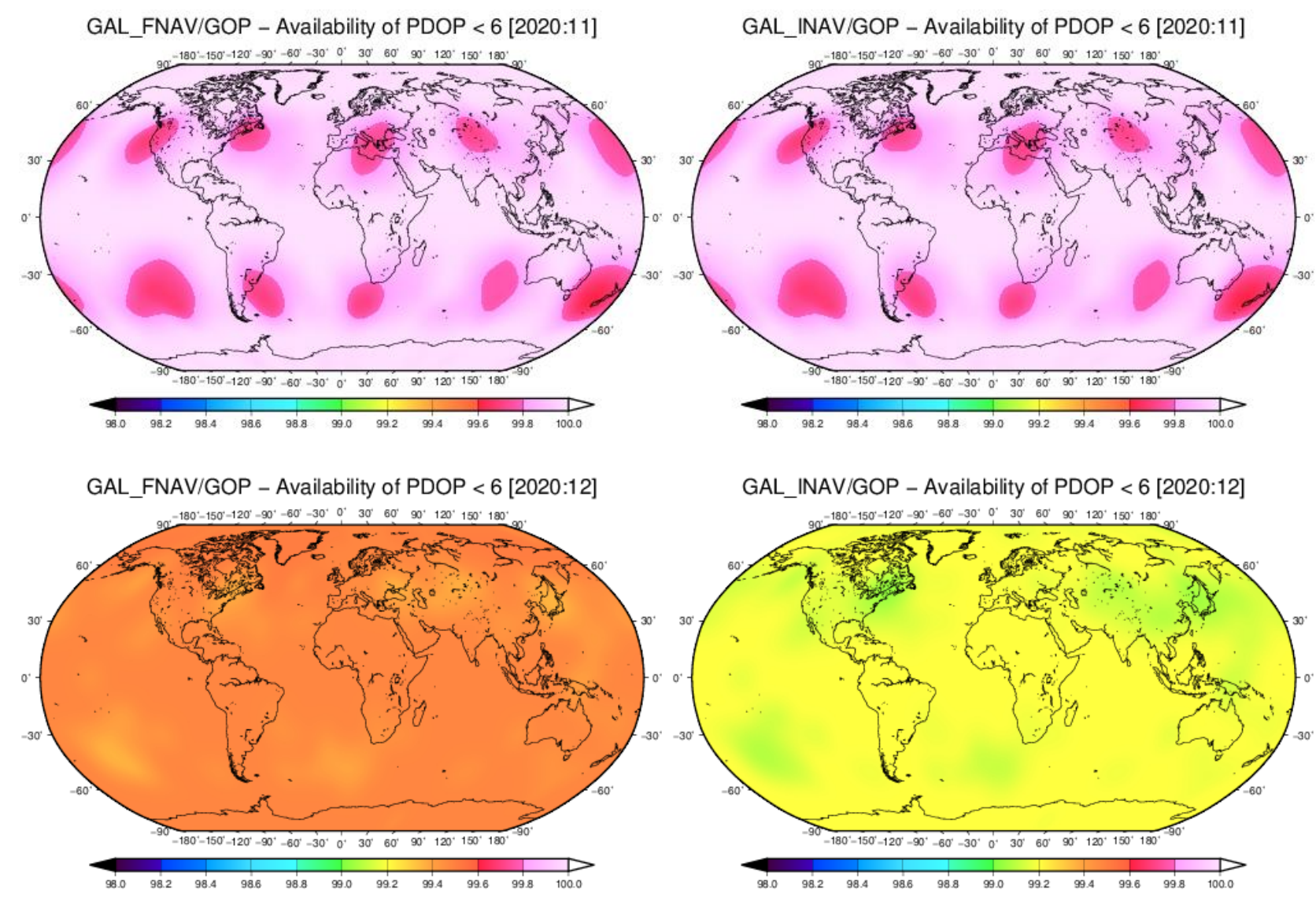

Fig. 7. Mean availability of PDOP<6 for November (top) and December (bottom) 2020 for Galileo FNAV (left) and INAV (right) navigation data types

\section{MEASURED POSITIONING PERFORMANCE}

The Galileo horizontal and vertical positioning performance is assessed at GOP using 14 selected sites distributed globally, and providing Galileo E1, E5a and E5b signals. First, the reference coordinates are estimated with the Precise Point Positioning (PPP, Zumberge et al. 1997) method on a daily basis, using the G-Nut/Geb software and GOP rapid products.

A Single Point Positioning (SPP) method is then performed on a daily basis using the GNut/Anubis software (Václavovic and Douša 2016), and code observations from the selected stations. Two independent positioning solutions exploit ionosphere-free linear combinations of $\mathrm{E} 1+\mathrm{E} 5 \mathrm{a}$ and $\mathrm{E} 1+\mathrm{E} 5 \mathrm{~b}$. The Galileo FNAV and INAV navigation type, both obtained from the GOP BRDC files, is used in the former and the latter solution. Considering the SHS, DVS, SISA, and the ephemeris validity period, all healthy satellites above the 5-degree elevation angle cut-off are used. Station coordinates, tropospheric path delay, and receiver clock offset are estimated epoch by epoch (if PDOP $<6$ is satisfied) at a $30-$ sec sampling interval.

Figure 8 displays time-series and histograms of horizontal and vertical positioning errors estimated at the station AREG and using E1+E5a and E1+E5b signals with FNAV and INAV navigation data, respectively. The horizontal error, absolute vertical error and PDOP are displayed in blue, green and yellow colours. 

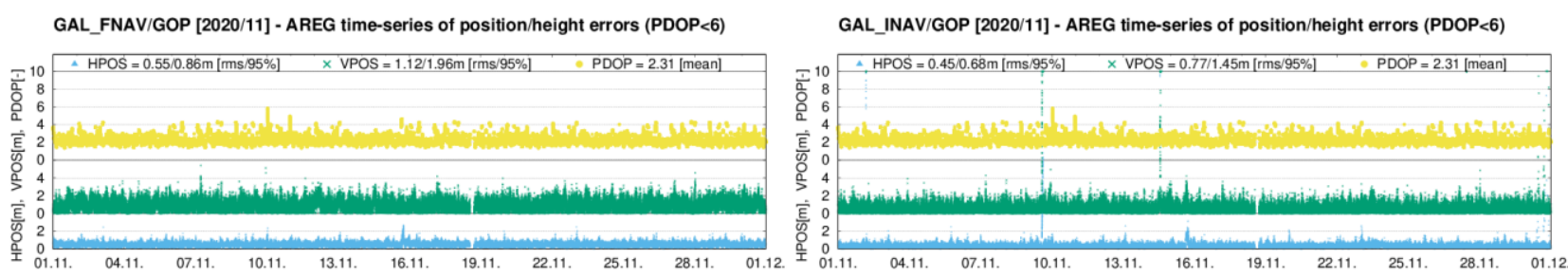

GAL_FNAV/GOP [2020/11] - AREG histograms of position/height errors (PDOP<6)
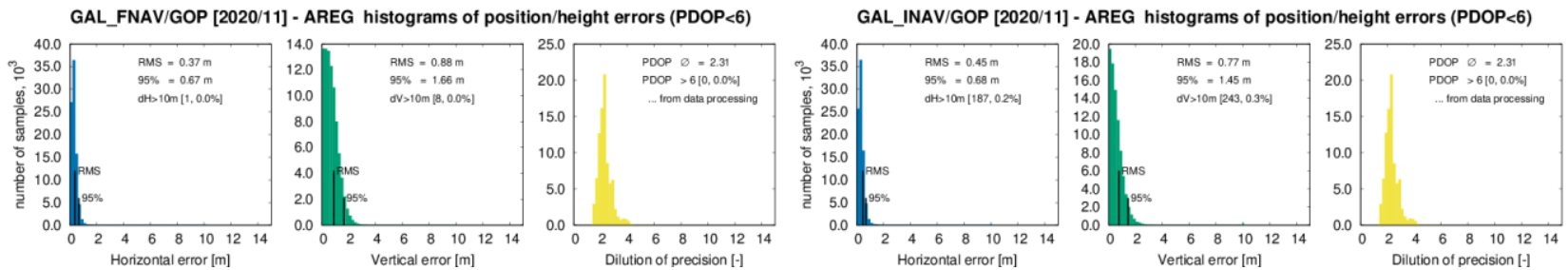

Fig. 8. Sample time-series (top) and histograms (bottom) of horizontal and vertical positioning errors using $\mathrm{E} 1+\mathrm{E} 5 \mathrm{a}$ with FNAV and E1+E5b with INAV solution at AREG stations in November, 2020

Finally, Table 1 shows a typical monthly performance of Galileo horizontal and vertical positioning errors (expressed in the $95^{\text {th }}$ percentiles). The best three global stations (AREG, METG, and YEL2) achieved $0.6-1.0 \mathrm{~m}$ and $1.3-1.8 \mathrm{~m}$ horizontal and vertical errors respectively, and the worst three stations (ASCG, JFNG, and MAYG) achieved 1.3-1.8m and $2.5-3.5 \mathrm{~m}$, respectively. The results were similar for solutions using FNAV and INAV types during 2019 and 2020 performing the best from all other GNSS constellations.

Table 1. $95^{\text {th }}$ percentiles of horizontal and absolute vertical positioning errors at 14 sites

\begin{tabular}{|c|c|c|c|c|c|c|c|c|c|c|c|c|}
\hline \multirow[b]{3}{*}{ Station } & \multicolumn{6}{|c|}{ 95\% HPE [m] } & \multicolumn{6}{|c|}{ 95\% VPE [m] } \\
\hline & \multicolumn{3}{|c|}{ E1+E5a / FNAV } & \multicolumn{3}{|c|}{ E1+E5b / INAV } & \multicolumn{3}{|c|}{ E1+E5a / FNAV } & \multicolumn{3}{|c|}{ E1+E5b / INAV } \\
\hline & Oct & Nov & Dec & Oct & Nov & Dec & Oct & Nov & Dec & Oct & Nov & Dec \\
\hline AREG & 67 & 67 & 4 & 0.68 & 0.68 & 0.65 & 66 & 1.66 & 1. & 1.46 & 1.45 & 1.48 \\
\hline ASC & 55 & 1.52 & 1.46 & 1.76 & 1.73 & 1.66 & 2.71 & 2.63 & 2. & 2.94 & 2.94 & 2.82 \\
\hline & .97 & 1.19 & 0.91 & 1.06 & 1.28 & 1.00 & .01 & 2.25 & 1.86 & 2.09 & 2.29 & 1.91 \\
\hline & .80 & 0 & - & 0.86 & 0.85 & 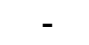 & .91 & & 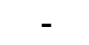 & 3 & 2.04 & - \\
\hline & 29 & & 1.4 & 1.47 & 1.4 & 1.4 & 2.73 & 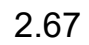 & 2. & 8 & 7 & 3.05 \\
\hline & 94 & 0.93 & $0 . \subseteq$ & 1.05 & 1.02 & 1.00 & .17 & 2.25 & 2.20 & 2.39 & 2.48 & 2.40 \\
\hline MA & 83 & 0.84 & 0.8 & 0.90 & 0.90 & 0.87 & 1.49 & 1.48 & 1.47 & 1.57 & 1.58 & 1.54 \\
\hline & & & & & & & & & & & & 2.77 \\
\hline & 88 & 0.90 & 0.9 & 0.98 & 1.00 & 1.01 & 1.26 & 1.29 & 1.32 & 1.35 & 1.39 & 1.37 \\
\hline OWI & 1.20 & 1.45 & 1.04 & 1.33 & 1.55 & 1.13 & 2.31 & 2.48 & 1.98 & 2.43 & 2.55 & 2.04 \\
\hline РОTS & 1.52 & 1.48 & 1.48 & 1.67 & 1.62 & 1. & 2.01 & 1.96 & 2.00 & 2.16 & 2.12 & 2.13 \\
\hline & & & 0. & & & 1. & 1.85 & & 1. & 1.94 & & 1.74 \\
\hline $\mathbf{U}$ & & 1. & 1. & 1.35 & 1.33 & 1. & 1.88 & 1.78 & 1. & 1.96 & 1.86 & 1.85 \\
\hline YEL2 & 0.86 & 0.78 & 0.86 & 0.95 & 0.84 & 0.93 & 1.55 & 1.41 & 1.51 & 1.63 & 1.50 & 1.58 \\
\hline Mean & 1.08 & 1.11 & 1.08 & 1.20 & 1.22 & 1.19 & 2.01 & 2.02 & 1.96 & 2.13 & 2.14 & 2.05 \\
\hline
\end{tabular}

\section{DISCUSSION}

The Galileo OS SIS performance monitoring at GOP is a valuable contribution to the Galileo Reference Centre as relaying on an independent chain of the processes using independent software and mandatory inputs. Additionally, the GOP solution is uniquely capable of operating in a rapid mode (or even shorter), i.e. within 1-2 days after last observations when based on the own fast consolidation of global navigation files, generation of GPS and Galileo reference products and calculated key-parameter indicators using the G-Nut software tools. 
During the entire period (2018/Q4 - 2020/Q4), the Galileo OS SIS performed excellently in term of aspects being monitored at GOP, and satisfied the Minimum Performance Level (MPL) defined in the OS SIS SDD document (GSA, 2019). However, several special events has been observed, analysed and reported to the GRC and GSA on a quarterly basis. These related to the positioning performance were particularly analysed:

- 2018, Nov 7-8 - unplanned discontinuity of navigation data for all the satellites over 27 hours (reported with NAGU2018027-028).

- 2019, Feb 11 - activating new Galileo satellites (E13, E15, E33 and E36), and firstly achieving 24-hour continuous Galileo PDOP < 6 in a global scope.

- 2019, July 11-17 - unplanned unavailability of OS SiS over 6 days for all satellites (reported with NAGU2019025-027).

- 2019, Sept 2-3 - satellite on-board navigation data provision only (i.e. without ground update) for all satellites over 12 hours.

- 2019, Oct 29 - short-term E11 satellite clock degradation (followed by the satellite deactivation) with a 30-min impact on positioning around the South-East Africa.

- 2020. Dec 14 - 3h and 5h provision of on-board navigation data for all satellites and FNAV and INAV, respectively.

- 2020, Oct 30 - activating the two eccentric Galileo satellites (E14 and E18).

\section{SUMMARY}

The Galileo system is going to provide various services to billions of users around the world, hence there is a strong need for a continuous performance monitoring of the service components. Since 2018, GOP has contributed to an independent monitoring of the Galileo Open Service Signal in Space performance, namely through the supporting of the Galileo Reference Centre and by providing reference station data quality control, rapid reference orbit and clock products, and key-parameter indicators with both a service volume monitoring and a site-specific measured positioning. The GOP has is principally able to extend its activity to additional KPIs, other GNSS systems or even to a real-time monitoring.

\section{REFERENCES}

Arnold, D., Meindl, M., Beutler, G, Dach R, Schaer S, Lutz S, Prange L, Sośnica K, Mervart M, Jäggi A (2015). CODE's new solar radiation pressure model for GNSS orbit determination. J. Geod. 89, 775-791, doi:10.1007/s00190-015-0814-4

Dach, R., Lutz, S., Walser, P., Fridez, P. (Eds) (2015) Bernese GNSS Software Version 5.2. User manual, Astronomical Institute, University of Bern, Bern Open Publishing. doi:10.7892/boris.72297

Dow, J.M., Neilan, R. E., and Rizos, C. (2009) The International GNSS Service in a changing landscape of Global Navigation Satellite Systems, J. Geod. 83:191-198, doi:10.1007/s00190-008-0300-3 
Douša, J. (2004) Precise Orbits for Ground-Based GPS Meteorology: Processing Strategy and Quality Assessment of the Orbits Determined at Geodetic Observatory Pecný, J. Meteor. Soc. Jap., 82:371-380

Douša, J. (2012) Development of the GLONASS ultra-rapid orbit determination at Geodetic Observatory Pecný, In: Geodesy for Planet Earth, Proceedings of the IAG Symposium, S.Kenyon, M.C.Pacino, U.Marti (eds.), Buenos Aires, Aug 31-Sep 4, IAG Symposia, Springer, 136:1029-1036

Douša, J. (2018) GOP's consolidated multi-GNSS navigation data archive. https://doi.org/10.24414/c4ba-kf16

Ge, M., Chen, J., Douša, J., Gendt, G., Wickert, J. (2012) A computationally efficient approach for estimating high-rate satellite clocks corrections in real-time, GPS Solut., Springer Berlin/Heidelberg, 16(1):9-17, doi:10.1007/s10291-011-0206-z

GSA (2019) GNSS Galileo Initial Services - Open Service SDD (Service Definition Document), Issue 1.1, May 2019

Loyer, S., Perosanz, F., Mercier, F., Capdeville, H., Marty, J.C. (2012) Zero-difference GPS ambiguity resolution at CNES-CLS IGS Analysis Center. J. Geod. 86:991-1003. doi:10.1007/s00190-012-0559-2

Montenbruck, O., Hauschiled, A., Steigenberger, P. (2014) Differential Code Bias Estimation using Multi-GNSS Observations and Global lonosphere Maps, Navig. J. ION, 61(3):191-201, doi:10.1002/navi.64

Montenbruck, O., Steigenberger, P., Prange, L., Deng, Z., Zhao, Q., Perosanz, F., Romero, I., Noll, C., Stürze, A., Weber, G., Schmid, R., MacLeod, K., Schaer, S. (2017) Multi-GNSS Experiment (MGEX) of the International GNSS Service (IGS) Achievements, Prospects and Challenges, Adv. Space Res. 59(7):1671-1697, doi:10.1016/j.asr.2017.01.011

Prange, L., Dach, R., Lutz, S., Schaer, S., Jäggi, A. (2015) The CODE MGEX Orbit and Clock Solution. In: Rizos C., Willis P. (eds) IAG 150 Years. IAG Symposia, Springer, Vol 143

Prange, L., Orliac, E., Dach, R. et al. (2017) CODE's five-system orbit and clock solutionthe challenges of multi-GNSS data analysis, J. Geod., 91:345

Zhao, L., Douša, J., Ye, S., Václavovic, P. (2020) A flexible strategy for handling the datum and initial bias in real-time GNSS satellite clock estimation, J. Geod., 94:1, doi:10.1007/s00190-019-01328-9

Zumberge, J.F., Heflin, M.B., Jefferson, D.C., Watkins, M.M., Webb, F.H. (1997) Precise point positioning for the efficient and robust analysis of GPS data from large networks J. Geoph. Res. (SE) 102:5005-5017

Václavovic, P., Douša, J. (2016) G-Nut/Anubis - open-source tool for multi-GNSS data monitoring, In: Rizos Ch and Willis P (eds), IAG Symposia Series, IAG 150 Years. Springer, 143:775-782, doi:10.1007/1345_2015_157 